\title{
The Properties of Epoxy Composites Containing Aligned Iron Oxide Nanorods by Low Magnetic Field
}

\author{
Yu-Ting Li ${ }^{1}$, Chuan-Guo Ma ${ }^{1,2, a, ~}{ }^{*}$, Qi-Ju Ou ${ }^{1}$, Hua-Mei Wan ${ }^{1}$, Pei-Bang Dai ${ }^{1,2}$ \\ ${ }^{1}$ School of Materials Science and Engineering, Guilin University of Electronic Technology, Guilin, \\ Guangxi, China \\ ${ }^{2}$ Guangxi Key Laboratory of Information Materials, Guilin, Guangxi, China \\ amachuanguo@guet.edu.cn \\ *Corresponding author
}

Keywords: $\mathrm{Fe}_{2} \mathrm{O}_{3}$ nanorods, Magnetic Field, Epoxy Resin, Fracture toughness.

\begin{abstract}
The magnetic iron oxide $\left(\mathrm{Fe}_{2} \mathrm{O}_{3}\right)$ nanorods were synthesized, and the epoxy resin (EP) composites with the content of $1 \mathrm{wt} \% \mathrm{Fe}_{2} \mathrm{O}_{3}$ were prepared in the condition of magnetic field. $\mathrm{X}$ ray diffraction, scanning electron microscopy and comprehensive physical properties measurement system were used to characterize the composition, morphology and magnetic properties of the nanorods, respectively. The effects of a low magnetic field $(\leq 0.06 \mathrm{~T})$ on fracture toughness, compressive strength and glass transition temperature of the composites were specifically investigated. The results show that the critical stress intensity factor, critical strain energy release rate and the compressive strength of $\mathrm{Fe}_{2} \mathrm{O}_{3} / \mathrm{EP}$ composites with magnetic field induction are 1.33 $\mathrm{MPa} \cdot \mathrm{m}^{1 / 2}, 0.56 \mathrm{KJ} / \mathrm{m}^{2}$ and $206.5 \mathrm{MPa}$, which are increased by $7.3 \%, 27 \%$ and $15 \%$ compared to of EP effectively.
\end{abstract}

\section{Introduction}

Epoxy resin (EP) is a kind of thermosetting resins with excellent mechanical properties and strong adhesive, which was widely used in the fields of coatings, adhesives, sports, ships, aerospace and others. However, the inherent high cross-linking of EP is easy to cause the brittle fracture of the products, which greatly limits the application of EP. According to the literature [1], the introduction of nanoparticles cause pinning, branching, bridging and deflection of crack, which results in decreasing of the crack driving force at the crack tip, thus plays an important role in improving the fracture toughness of EP. In addition, Khanet al. [2] have indicated that the nanoparticles with large aspect ratio (lamellar, one-dimensional rod like) are favorable for the bridging and deflection of the crack, and the ordered arrangement of the nanorods perpendicular to the direction of crack growth further promotes the crack bridging and deflection. Even though there are many methods to make the orientation of nanoparticles, the magnetic field (MF) induced orientation method is more simple and efficient [3]. Wu et al. [4] have loaded $\mathrm{Fe}_{3} \mathrm{O}_{4}$ onto graphene nanoplates (GNS) and prepared the GNS - $\mathrm{Fe}_{3} \mathrm{O}_{4} / \mathrm{EP}$ composites with and without MF. The results show that when the content of $\mathrm{GNS}-\mathrm{Fe}_{3} \mathrm{O}_{4}$ was $1 \mathrm{wt} \%$, the critical energy release rate $\left(G_{I C}\right)$ of composites with MF is $819 \mathrm{~J} / \mathrm{m}^{2}$, which is $511 \%$ higher than EP and 50\% higher than that without MF. Ma et al.[5] have studied the influence of nickel particle-loaded carbon nanotubes (CNTs) on fracture toughness of magnetic nanocomposites with MF. The results show that when CNTs content was $1 \mathrm{wt} \%$, the critical stress intensity factor $\left(\mathrm{K}_{\mathrm{IC}}\right)$ and $\mathrm{G}_{\mathrm{IC}}$ of composites with MF were increased by $49 \%$ and $120 \%$ compared to EP and increased by $12 \%$ and $25 \%$ compared to the composites without MF. From the previous literatures, the orientation of lamellar or rod-like particles under MF has to require help of magnetic particles, which makes the process more complicated and inefficient. So far, few studies have been conducted to improve the fracture toughness of EP by one-dimensional magnetic nanoparticles (eg. $\mathrm{Fe}_{2} \mathrm{O}_{3}$ nanorods) with $\mathrm{MF}$ induction. The $\mathrm{Fe}_{2} \mathrm{O}_{3}$ nanorods, which were dehydrated by iron oxyhydroxide $(\mathrm{FeOOH})$ particels, not only maintain the rod-like morphology of $\mathrm{FeOOH}$, but also have good magnetic properties to be aligned under a low $\mathrm{MF}(\leq 0.1 \mathrm{~T})$. In this work, the $\mathrm{Fe}_{2} \mathrm{O}_{3}$ nanorods were prepared by two-step method, the $\mathrm{Fe}_{2} \mathrm{O}_{3} / \mathrm{EP}$ composites were prepared without MF and with MF. The influence of the aligned 
nanorods on the fracture properties, compressive properties and glass transition temperature $\left(\mathrm{T}_{\mathrm{g}}\right)$ of the composites were investigated when the $\mathrm{Fe}_{2} \mathrm{O}_{3}$ nanorods content was $1 \mathrm{wt} \%$.

\section{Experimental}

\section{Materials}

Ferrous chloride tetrahydrate $\left(\mathrm{FeCl}_{2} \cdot 4 \mathrm{H}_{2} \mathrm{O}\right)$ and sodium hydroxide $(\mathrm{NaOH})$ were purchased from Xilong chemical Limited by Share Ltd (China). Epoxy resin (diglycidyl ether of bisphenol A, RIMR 135), and curing agent (modified amine, RIMH 134) were supplied by Hexion Specialty Chemicals Inc (USA).

\section{Preparation of $\mathrm{Fe}_{2} \mathrm{O}_{3} / \mathrm{EP}$ Composites}

The $\mathrm{Fe}_{2} \mathrm{O}_{3}$ nanorods were obtained by the two-step method in the literature [6], and the uniform mixture of $\mathrm{Fe}_{2} \mathrm{O}_{3}$ and EP was obtained by solvent dispersion method. After the mixture and curing agent ( $30 \%$ of EP by weight), were mixed, evenly, in a vacuum oven and then poured into the mold under MF (0.06T) as shown in Fig. 1 and without MF as control. The $\mathrm{Fe}_{2} \mathrm{O}_{3} / \mathrm{EP}$ composites with and without MF can be obtained after curried at $60^{\circ} \mathrm{C}$ for 12 hours. In this study, a single edge notch specimen was fabricated according to ASTM D5045-99 standard.

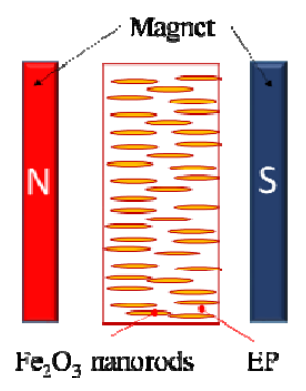

Figure 1. A schematic representation of preparation of $\mathrm{Fe} 2 \mathrm{O} 3 / \mathrm{EP}$ composites under MF .

\section{Characterization}

The composition of $\mathrm{Fe}_{2} \mathrm{O}_{3}$ nanorods was analyzed by $\mathrm{X}$ ray diffraction (XRD, model D8-2-Advance, Germany BRUKER). The morphology of $\mathrm{Fe}_{2} \mathrm{O}_{3}$ nanorods and composite section were investigated using scanning electron microscopy (SEM, model Quanta450FEG, US FEI). The integrated physical properties measurement system (PPMS, model PPMS-9, US Quantum Design) was used to examine the magnetic properties of $\mathrm{Fe}_{2} \mathrm{O}_{3}$ nanorods at room temperature. The mechanical properties of composites were measured on the universal material testing machine (WDW-E100, Ji'nan Shijin Group Co. Ltd.) with a crosshead speed of $1.0 \mathrm{~mm} / \mathrm{min}$. The specimens for compression test were prepared according to GBT2567-2008. $\mathrm{K}_{\mathrm{IC}}$ was determined using a single edge notch bend (SENB) test according to ASTM D5045-99 standard and the modified SENB specimens [5]. The $\mathrm{T}_{\mathrm{g}}$ of composite materials was tested by differential scanning calorimetry (DSC, DSC204, NETZSCH) at the temperature range of -40 to $200^{\circ} \mathrm{C}$ and with a heating rate of $10 \mathrm{~K} / \mathrm{min}$.

\section{Results and Discussion}

\section{Characterization of $\mathrm{Fe}_{2} \mathrm{O}_{3} \mathrm{Nanorods}$}

Fig.2 (a) is the XRD pattern of Fe2O3nanorods. According to the XRD data analysis software (Jade 6.5), the diffraction peaks at $45.62^{\circ}, 51.43^{\circ}, 60.64^{\circ}, 77.72^{\circ}$ and $84.75^{\circ}$ correspond to the crystal plane (400), (511), (440) of the standard Fe2O3 (PDF \# 39-1346) respectively. In addition, the other peaks are not observed in the pattern, which reveals that the product is pure Fe2O3.As shown in Fig. 2 (b), Fe2O3 has a one-dimensional rod shape, the length is about $0.6 \mu \mathrm{m}$ and the 
diameter is about 50nm, all of nanorods are uniform. Fig. 2 (c) and (d) show the magnetic properties of Fe2O3nanorods. It can be seen from the Fig. (c), the Fe2O3nanorods show a high saturation magnetization of $38.06 \mathrm{emu} / \mathrm{g}$ and paramagnetism. Fig. 2 (d) is the SEM image of Fe2O3 dispersed in alcohol and induced by MF. It can be found that not only the aggregated nanorods but also the single $\mathrm{Fe} 2 \mathrm{O} 3$ is aligned along the direction of the magnetic field. This indicates that the induction of MF could lead the Fe2O3nanorods to be oriented.
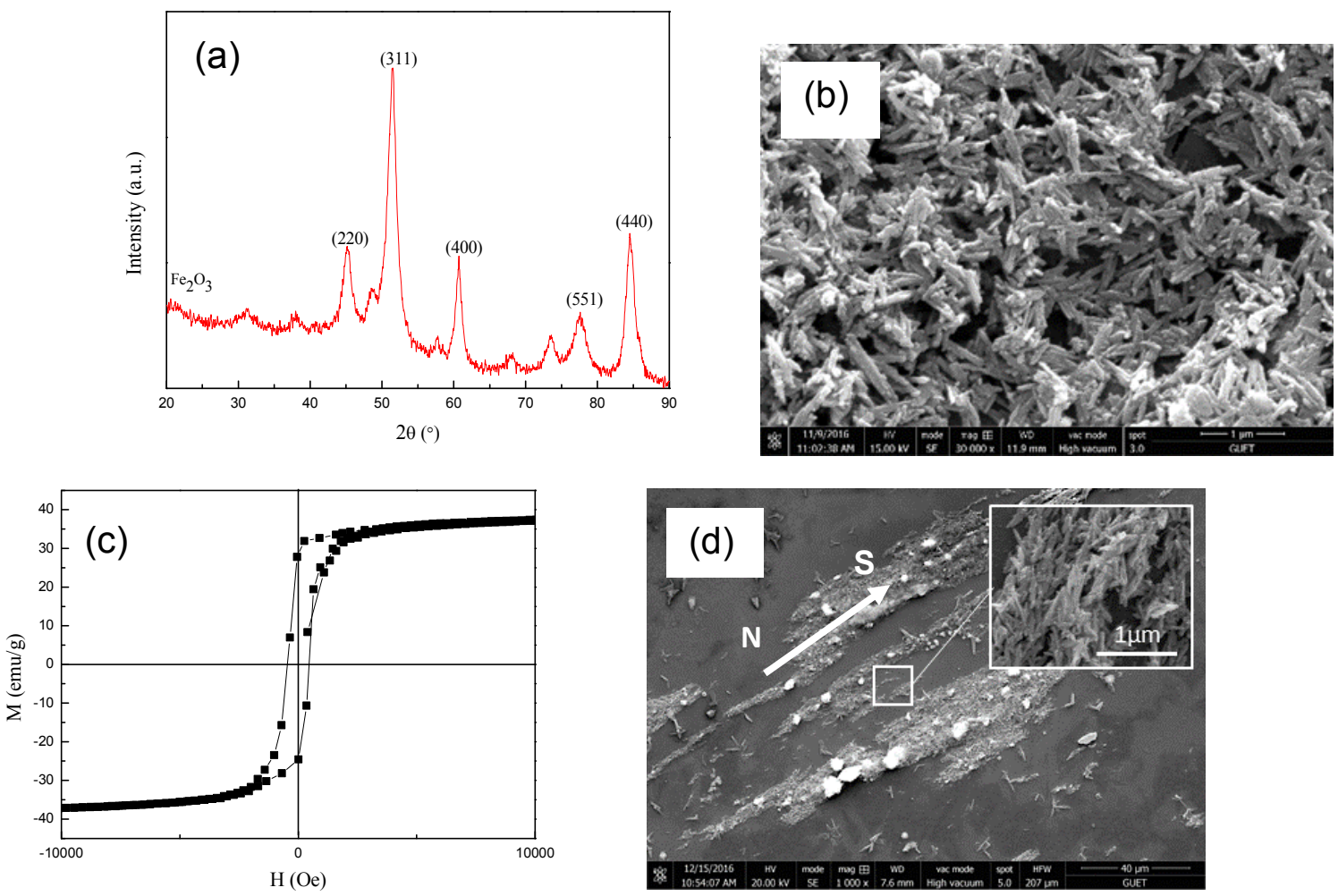

Figure 2. Characterization of Fe2O3nanorods. (a) XRD pattern, (b) SEM image without MF induction, (c) hysteresis loop and (d) SEM image with MF induction .

\section{Characterization of $\mathrm{Fe}_{2} \mathrm{O}_{3} / \mathrm{EP}$ Composites}

\section{Fracture Toughness}

Table 1. Mechanical properties of composites.

\begin{tabular}{cccc}
\hline Materials & $\mathrm{K}_{\mathrm{IC}}\left(\mathrm{MPa} \cdot \mathrm{m}^{1 / 2}\right)$ & $\mathrm{G}_{\mathrm{IC}}\left(\mathrm{KJ} / \mathrm{m}^{2}\right)$ & Compressive strength $(\mathrm{MPa})$ \\
\hline EP & 1.24 & 0.44 & 179 \\
$\mathrm{Fe}_{2} \mathrm{O}_{3} /$ EP without MF & 1.04 & 0.31 & 207.5 \\
$\mathrm{Fe}_{2} \mathrm{O}_{3} /$ EP with MF & 1.33 & 0.56 & 206.5 \\
\hline
\end{tabular}

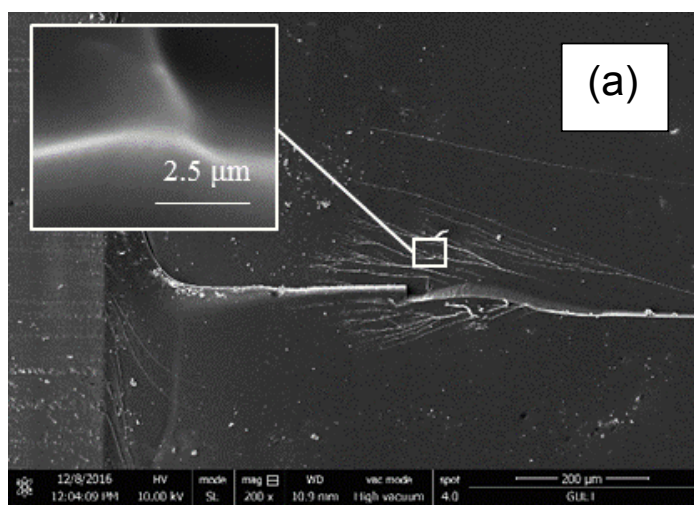



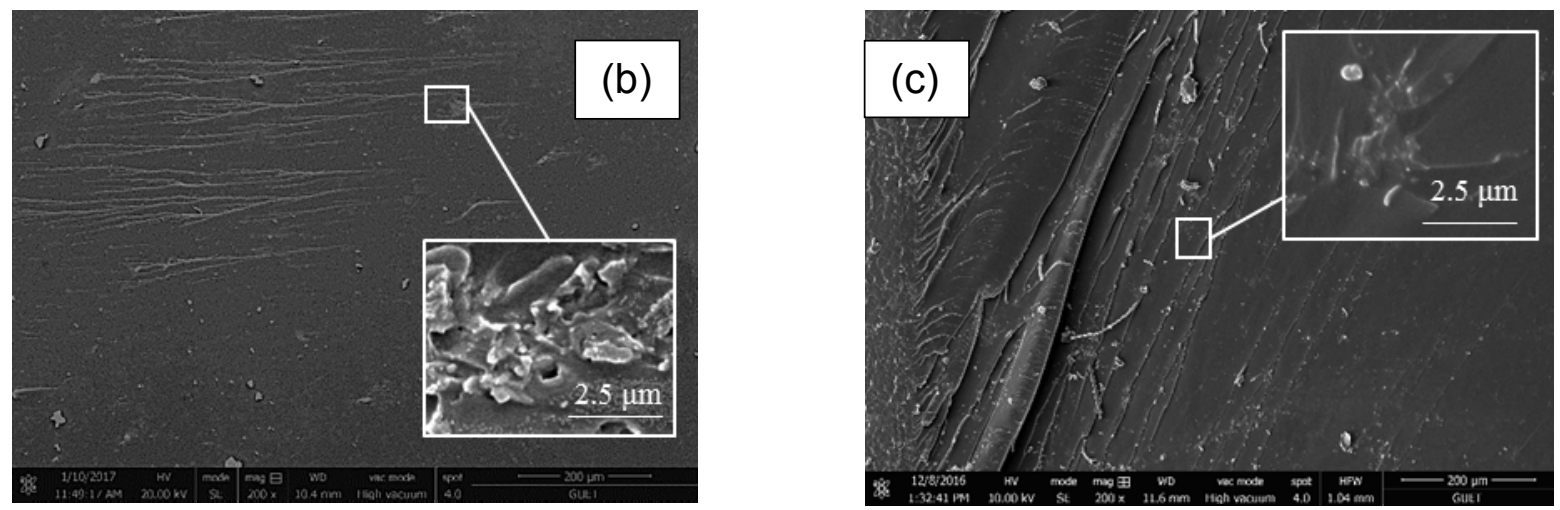

Figure3. Fracture morphology of composites. (a) EP, (b) Fe2O3/EP composites without MF and (c) Fe2O3 /EP composites with MF.

Table 1 shows the fracture toughness of the composites with MF and without MF by $\mathrm{K}_{\mathrm{IC}}$ and $\mathrm{G}_{\mathrm{IC}}$. From the data, it can be seen that the $\mathrm{K}_{\mathrm{IC}}$ and $\mathrm{G}_{\mathrm{IC}}$ of the composites without MF are $1.04 \mathrm{MPa} \cdot \mathrm{m}^{1 / 2}$ and $0.31 \mathrm{KJ} / \mathrm{m}^{2}$, which are lower than those of the pure EP at $1.24 \mathrm{MPa} \cdot \mathrm{m}^{1 / 2}$ and $0.44 \mathrm{KJ} / \mathrm{m}^{2}$. Whereas, the $\mathrm{K}_{\mathrm{IC}}$ and $\mathrm{G}_{\mathrm{IC}}$ of the composites with $\mathrm{MF}$ are $1.33 \mathrm{MPa} \cdot \mathrm{m}^{1 / 2}$ and $0.56 \mathrm{KJ} / \mathrm{m}^{2}$, which are $7.3 \%$ and $27 \%$ higher than that of pure EP, respectively. In terms of the improvement in $\mathrm{K}_{\mathrm{IC}}$ and $\mathrm{G}_{\mathrm{IC}}$ compared to that of the pure EP, the results is not particularly significant compared to the reported results. Taoet al.[7] reported that the $\mathrm{K}_{\mathrm{IC}}$ of the composites is lower than that of pure EP when adding the same amount of $\mathrm{Fe}_{2} \mathrm{O}_{3}$ particles in the EP, which is similar to that of the composites without MF. However the percentage of increase in $\mathrm{K}_{\mathrm{IC}}$ and $\mathrm{G}_{\mathrm{IC}}$ of the composites with MF is lower than that in the work[5]. It may be mainly attributed to the poor dispersion of the nanorods in the composites and weak interface interaction between the nanorods and the EP, which are shown in the next fracture surface morphology analysis. But it is noted that the $\mathrm{K}_{\mathrm{IC}}$ and $\mathrm{G}_{\mathrm{IC}}$ of the composites with $\mathrm{MF}$ are $27.9 \%$ and $80.6 \%$ higher than that of the composites without MF, respectively. This indicates that the effect of MF on the properties of the composites is very significant even compared with the reported results [5].

In order to further explain the toughening effect of Fe2O3nanorods on EP, the fracture surface morphology of composites was analyzed by SEM. Fig. 3 (a) is the fracture surface of pure EP, which can be seen that the crack tip is smooth and without any other material. The fracture surface of the composites without MF shown in Fig. 3 (b), as can be seen that at the beginning of the crack has obvious nanoparticles and voids, which is due to the broken nanorods and pull out of nanorods when the composites broken. The behaviors of the nanorods could have led the energy to dissipate and play a role in improving the fracture properties of EP. Unfortunately, due to the interface defects between nanoparticles and EP, and the randomly dispersed nanoparticles in the composite without MF, the fracture of composites caused much more debonding of nanorods rather than the broken nanorods and pull-out of nanorods, does not achieve the purpose of toughening. This mechanism is illustrated in Fig. 4 (a). Fig. 3 (c) is the fracture surface of the composites with MF, which can be clearly seen that there are broken traces of the nanorods. With the help of MF induction, the nanorods are arranged perpendicular to the crack growth direction, and formed a continuous acicular network in EP. Under the action of stress, the crack has to pass all nanorods to break the composites as shown in Fig. 4 (b). The process is accompanied by more broken nanorods and pull out of nanorods. In addition, there is enough power to make the crack deflected or terminated, so as to improve the fracture toughness of EP. The toughening mechanism is the same as that proposed in the literature [1]. 

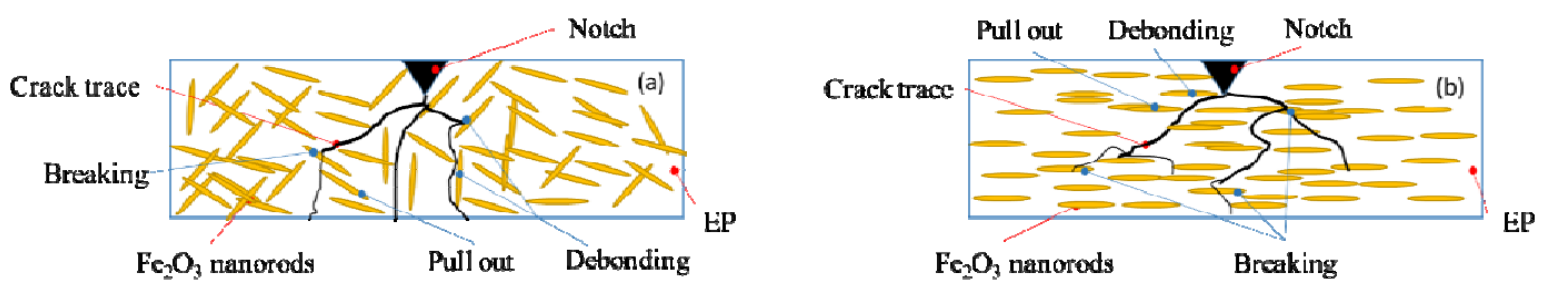

Figure4. Schematic diagram of Fe2O3nanorods toughening EP. (a)Without MF and (b) with MF.

\section{Compression Property and $T_{\mathrm{g}}$ of Composites}

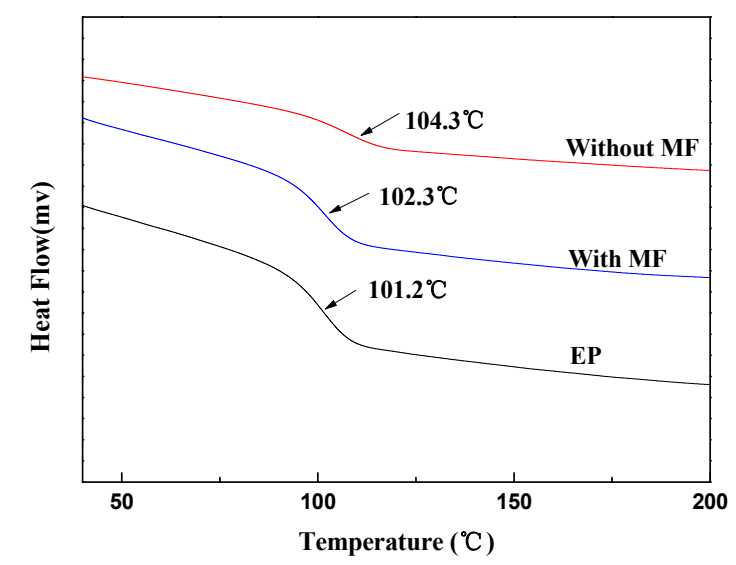

Figure 5.Tg of pure EP and the composites with and without MF

When the material is subjected to stress, besides the fracture failure, often accompanied by compression failure. As can be seen from Table 1, the compressive strength of $\mathrm{Fe}_{2} \mathrm{O}_{3} / \mathrm{EP}$ composites without MF is $207.5 \mathrm{MPa}$, which is $15.9 \%$ higher than that of pure EP. The compressive strength of composites with MF is $206.5 \mathrm{MPa}$, which is $15.4 \%$ higher than that of EP. Obviously, the addition of nanorods could improve the compressive properties of EP. In this work, the direction of the magnetic field is parallel to the direction of the compressive stress, which makes the rod-like particles parallel to the stress direction. Thus with the condition of the same load, the nanorods with MF are easier to be damaged than those without MF, so the compressive strength of composites without MF is slightly higher than that with MF.

The Tg of Fe2O3/EP composites were investigated by DSC. It is shown in Fig. 5 that the Tg of pure $\mathrm{EP}$ and $\mathrm{Fe} 2 \mathrm{O} 3 / \mathrm{EP}$ composites without $\mathrm{MF}$ and with $\mathrm{MF}$ are $101.2^{\circ} \mathrm{C}, 104.3^{\circ} \mathrm{C}$ and $102.3^{\circ} \mathrm{C}$, respectively. There are a lot of factors affecting the $\mathrm{Tg}$ of composites. In the composites the introduction of $\mathrm{Fe} 2 \mathrm{O} 3$, which hindered the movement of the EP molecule segment, makes the Tg of EP increased. From Fig. 2 (d), it can be seen that the Fe2O3nanorodsare easy to be gathered in the condition of MF. There may be a vacancy in the vicinity of the oriented nanorods, which cannot more efficiently restrict EP molecule segment compared to the randomly dispersed Fe2O3nanorods without MF. So the Tg of Fe2O3/EP composites without MF is higher than that with MF.

\section{Conclusions}

The magnetic Fe2O3nanorodswere successfully synthesized through two-step methodand can be aligned along the direction of the magnetic field. In addition, the Fe2O3/EP composites without MF and with MF were prepared when the content of Fe2O3 nanorods was $1 \mathrm{wt} \%$. The effects of MF on fracture toughness, compressive strength and Tg of the composites were compared with pure EP, respectively. Although the toughening effect is not particularly significant compared to the other 
works, the fracture toughness of $\mathrm{Fe} 2 \mathrm{O} 3 / \mathrm{EP}$ composites with MF is much higher than the composites without MF. It is revealed that the aligned $\mathrm{Fe} 2 \mathrm{O} 3$ nanorods can improve the fracture properties of EP effectively using a low MF. Furthermore, due to the addition of $\mathrm{Fe} 2 \mathrm{O} 3$ nanorods, the compressive strength and $\mathrm{Tg}$ of EP have been increased to a certain degree, no matter in the condition of MF or without MF.

\section{Acknowledgements}

The authors gratefully acknowledge the supports from the National Natural Science Foundation of China (Grant \#51363003), and the Guangxi Natural Science Foundation(2016JJA160040).

\section{References}

[1] H.Y. Liu, G.T. Wang, Y.W. Mai, On fracture toughness of nano-particle modified epoxy, J. Composites Part B Engineering, 42 (2011) 2170-2175.

[2] S.U. Khan, J.R. Pothnis, J.K. Kim, Effects of carbon nanotube alignment on electrical and mechanical properties of epoxy nanocomposites, J. Composites Part A Applied Science \& Manufacturing, 49 (2013) 26-34.

[3] S. Wu, R.B. Ladani, J. Zhang, Aligning multilayer graphene flakes with an external electric field to improve multifunctional properties of epoxy nanocomposites, J. Carbon, 94 (2015) 607-618.

[4] S. Wu, J. Zhang, R.B. Ladani, A novel route for tethering graphene with iron oxide and its magnetic field alignment in polymer nanocomposites, J. Polymer, 97 (2016) 273-284.

[5] C. Ma, H.Y. Liu, X. Du, Fracture resistance, thermal and electrical properties of epoxy composites containing aligned carbon nanotubes by low magnetic field, J. Composites Science \& Technology, 114 (2015) 126-135.

[6] C. Sudakar, G.N. Subbanna, T.R.N. Kutty, Effect of anions on the phase stability of $\gamma-\mathrm{FeOOH}$ nanoparticles and the magnetic properties of gamma-ferric oxide derived from lepidocrocite, J. Journal of Physics \& Chemistry of Solids, 64 (2003) 2337-2349.

[7] T. Sun, H. Fan, Z. Wang, X. Liu, Z. Wu, Modified nano $\mathrm{Fe}_{2} \mathrm{O}_{3}$-epoxy composite with enhanced mechanical properties, J. Materials \& Design, 87 (2015) 10-16. 